\title{
Magnetic field evolution of active regions and sunspots in connection with chromospheric and coronal activities
}

\author{
Toshifumi Shimizu ${ }^{1}$ \\ ${ }^{1}$ Institute of Space and Astronautical Science, Japan Aerospace Exploration Agency, \\ 3-1-1 Yoshinodai, Chuo, Sagamihara, Kanagawa 252-5210, Japan \\ email: shimizu@solar.isas.jaxa.jp
}

\begin{abstract}
Ca II H imaging observations by the Hinode Solar Optical Telescope (SOT) have revealed that the chromosphere is extremely dynamic and that ejections and jets are well observed in moat region around sunspots. X-ray and EUV observations show frequent occurrence of microflaring activities around sunspots; small emerging flux or moving magnetic features approaching opposite pre-existing magnetic flux can be identified on the footpoints for half of microflares studied, while no encounters of opposite polarities are observed at footpoints for the others even with SOT high spatial magnetorams (Kano et al. 2010). Another observations tell the involvement of twisted magnetic fields in the microflares accompanied by no polarity encounters at the footpoints. Some type of sunspot light bridges shows recurrent occurrence of chromospheric ejections, and photospheric vector magnetic field data suggests that twsited magnetic flux tubes lying along light bridge play vital roles in producing such ejections (Shimizu et al. 2009). This presentation reviewed observational findings from these studies. We will need to understand the 3D configuration of magnetic fields for better understanding of activity triggers in the solar atmosphere.
\end{abstract}

Keywords. Sun: activity, sun: chromosphere, sun: corona, sun: evolution, sun: magnetic fields, sun: photosphere, sunspots

\section{Introduction}

Soft X-ray and EUV images of the Sun show that intense X-ray and EUV emissions are mostly concentrated in active regions. The active region corona consists of a lot of loop-like structures, which trace magnetic field bundles filled with hot plasma in the corona. Bright loops are mainly rooted around penumbra of sunspots and nearby areas. It is dark in X-rays above umbra of sunspots, although the umbra is filled with strong magnetic flux.

The time series of soft X-ray and EUV images reveal that weak transient brightenings of small coronal loops, i.e., microflares, are frequently produced in the active region corona. The released energy of each brightening is in order of $10^{24}$ to $10^{28} \mathrm{ergs}$, which is about 6 orders of magnitude smaller than that of solar flares (Shimizu 1995). The number of flares has a power-law distribution as a function of energy, which continues to much small energy range, at least down to $10^{24} \mathrm{erg}$ (Aschwanden et al. 2000).

The Hinode Solar Optical telescope (SOT) has provided new views in the chromosphere, which is the interface layer between the photosphere and corona. The chromosphere observed with Ca II H line is much more dynamic than we have observed before. Plasma ejections and heating events are always observed around sunspots. This view can be seen in the Ca II H movies which capture sunspots and nearby areas located near the solar limb. 
One of outstanding questions is what is origins of activities in the corona and chromosphere. The magnetic energy stored in the atmosphere is used for causing activities. Magnetic reconnection is believed as the converter from magnetic energy to kinetic and thermal energy, but details of magnetic reconnection is still poorly understood. We do not exactly understand magnetic configuration observed around the activities, which is essential to understand what triggers magnetic reconnection. How such magnetic configuration can be created in the solar atmosphere? Key information for the understanding is observations of magnetic fields well combined with observations of activities.

\section{Magnetic Field Evolution in Active Regions}

Magnetic field evolution observed in active region NOAA 11039 was discussed in this section. This active region was well observed with SOT. Ca II H images and Na D longitudinal magnetograms were continuously acquired every 3 minutes in a long period from 29 December 2009 until 2 January 2010, with very limited number of short interruptions during the period. It is almost the first time for SOT to continuously monitor an active region for such a long period and this unique observation was realized during the year-end and new-year holiday period. The field of view covered almost the entire area of the active region. The movie observation successfully captured time evolution of several sunspots, including the birth of sunspots, the evolution of well-developed sunspots, and the decay of sunspots.

Fig. 1 is the zoom up of the area where the successive emergences of magnetic flux form a pair of sunspots. In between the newly forming positive-polarity sunspot and

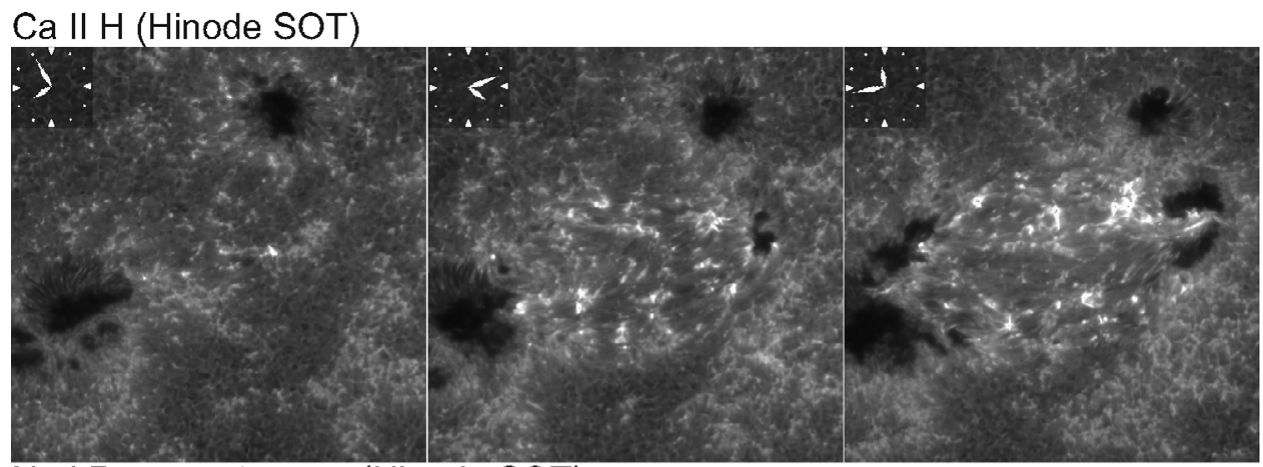

$\mathrm{Na}$ I D magnetogram (Hinode SOT)

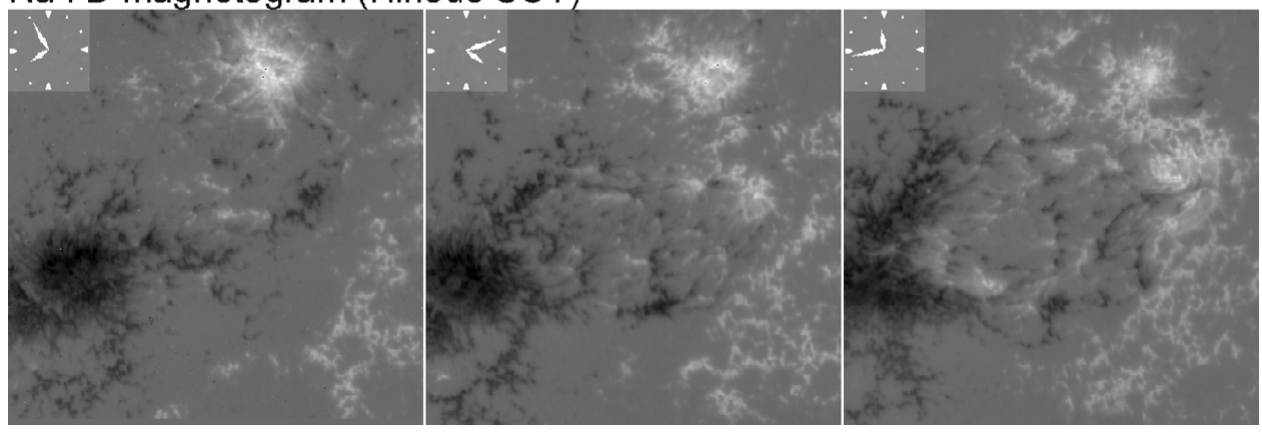

Figure 1. An emerging flux region appeared in NOAA 11039. Three snapshots were picked up from the continuous measurement of Ca II H (upper figures) and $\mathrm{Na}$ I D magnetogram (lower figures). Times are, from the left, 7:54, 16:12, and 23:44 UT on 30 December 2009. The field of view is 55.8 arcsec. 
the following negative-polarity sunspot, a lot of small magnetic bipoles are emerged. Cancellation of opposite polarities may be observed when one polarity flux meets the opposite polarity flux of another emerging dipole. Transient brightenings (bright, whitecolored patches in the figure) are observed in Ca II H above the area where the opposite polarities are in contact. They mean transient heating of chromospheric gas. Also, in many cases, microflaring activities of coronal loops may be observed in the corona. Bright transient brightenings in Ca II H mostly corresponds to the footprints of microflaring coronal loops. Merging of same polarities are also observed, which finally results in the development of sunspots at the both ends of the emerging flux region.

Fig. 2 shows the time evolution of a well-developed sunspot. Well-known, moving magnetic features, MMFs, are observed around the sunspot. They are small flux patches moving out from the outer edge of the penumbra in the radial direction. Some type of MMFs appears to be responsible for removing the magnetic flux from sunspots. The zone where MMFs are observed is called "moat." In the moat region, small flux emergences may be found, which is the major source for causing microflares around sunspots, as discussed in the next section. Cancelation of opposite polarities may be observed at the end of the moat.

Fig. 3 shows the disappearance of a sunspot. It is well observed that the concentrated sunspot flux is gradually fragmented. A sunspot light bridge may be observed when a sunspot is divided into two large segments. Ca II H movie shows less number of transient brightenings.
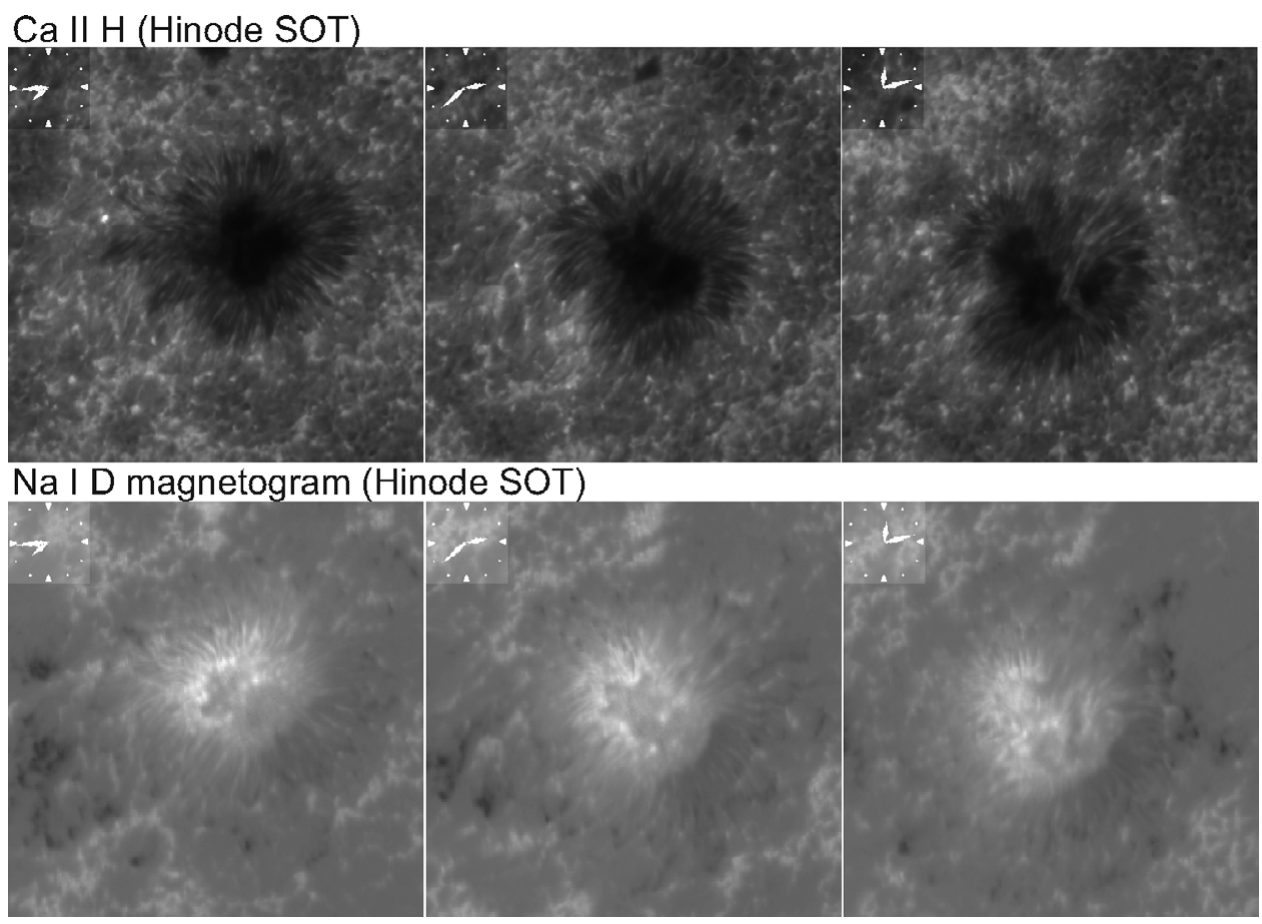

Figure 2. Evolution of a large sunspot developed in NOAA 11039. Three snapshots were picked up from the continuous measurement of Ca II H (upper figures) and Na I D magnetogram (lower figures). Times are, from the left, 7:44 and 14:38 UT on 1 January and 0:12 on 2 January 2010. The field of view is 55.8 arcsec. 


\section{Magnetic origins of microflares}

Photospheric magnetic activities triggering X-ray microflares were studied by Kano et al. (2010). They identified 55 microflares around a well-developed sunspot surrounded by a moat with high-cadence X-ray images from the Hinode X-ray Telescope, and searched for their photospheric counterparts in high spatial resolution line-of-sight magnetograms taken by the SOT. They found opposite magnetic polarities encountering each other around the footpoints of 28 microflares (half of the samples), while such encounters could not be found around the footpoints in the rest of microlfares, even though we examined high quality and high resolution SOT magnetograms.

It is also identified that emerging magnetic fluxes in the moat were the dominant origin for causing the encounters of opposite polarities (21 of 28 microflares). Note that this result supports results from La Palma - Yohkoh coordinated observations (Shimizu et al. 2002). Additionally, uni-polar moving magnetic features with the polarity same as the sunspot definitely caused the encounters of opposite polarities for 5 cases. For these two types of microflares, the observational results lead to two magnetic configurations including magnetic reconnection, which can explain naturally most of observational signatures.

When a small-scale flux is emerged from below the photosphere, we may observe X-ray and Ca II H brightenings. X-ray brightening as well as Ca II H brightening are observed almost above the contact where a polarity patch of the emergence is approaching the pre-existing magnetic flux with the opposite polarity. At that time, we may observe that
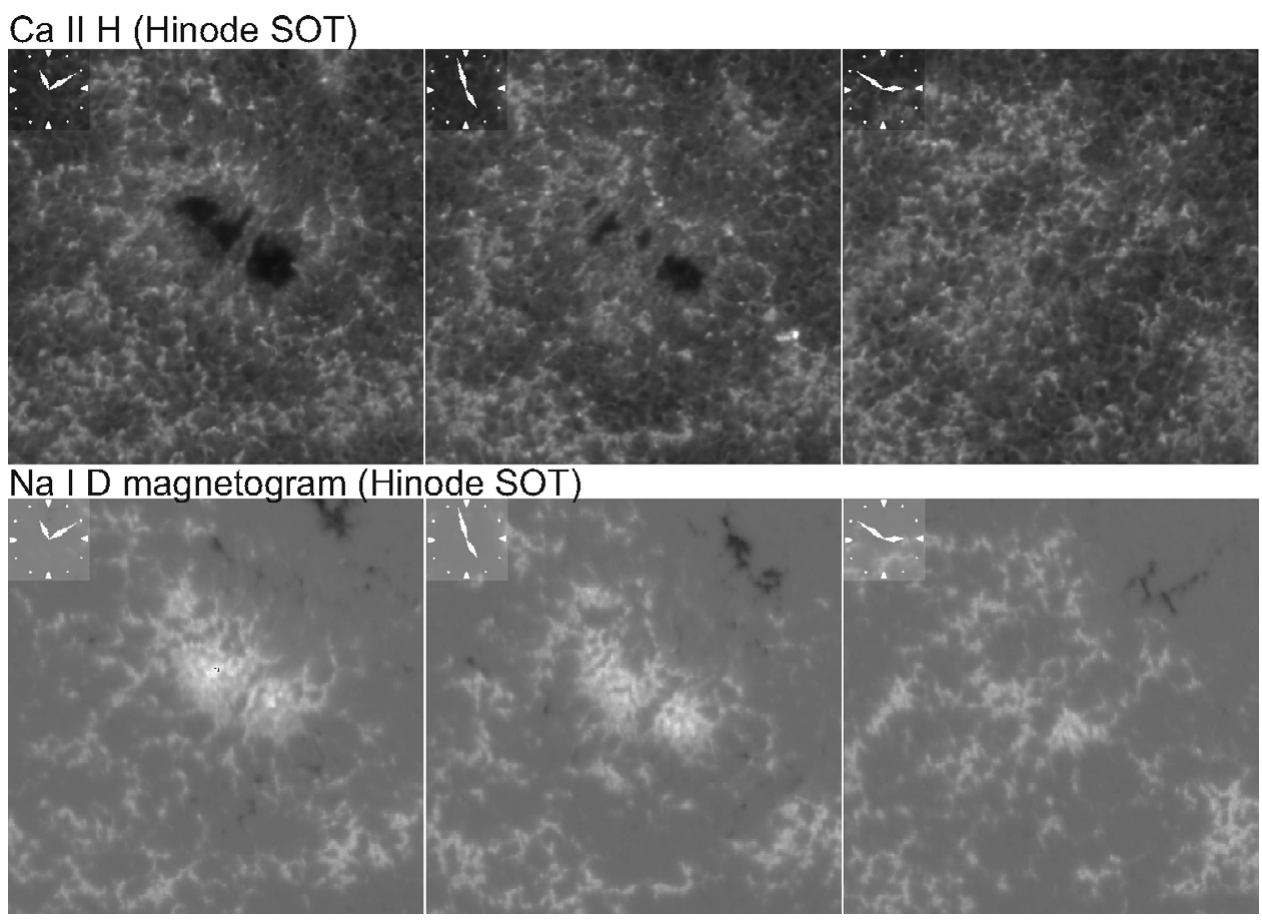

Figure 3. Disappearance of a sunspot observed in NOAA 11039. Three snapshots were picked up from the continuous measurement of Ca II H (upper figures) and Na I D magnetogram (lower figures). Times are, from the left, 23:09 on 29 December, 4:58 and 14:51 UT on 30 December 2009. The field of view is 55.8 arcsec. 
the magnetic flux is decreased after the occurrence of the microflare. The observations suggest that the emergence of magnetic flux makes a field discontinuity (current sheet) with the pre-existing field, causing magnetic reconnection in the current sheet as a rapid converter of magnetic energy to thermal and kinetic energies. Submergence of fields as a result of reconnection is observed as the decrease of magnetic flux.

When a small magnetic flux patch is detached from the penumbral outer edge of sunspots and moves outward as a uni-polar moving magnetic feature, it may make an approach to the opposite polarity patches which are sometimes seen in some portions of the moat outer edge. A faint loop-like microflare may be observed with such approach, and one end of the brightening loop is located at the encounter of the polarities. With occurring a microflare, we may observe that the magnetic flux is reduced with time. The observations suggest that the approaching flux makes a field discontinuity with the opposite flux, which results in magnetic reconnection in the discontinuity, although the released energy may be small.

\section{Twisted magnetic fields for creating activities}

About half of microflares did not show any encounters of opposite polarities, even when high spatial resolution magnetograms were examined. According to a preliminary study of such microflares observed on 1 May 2007, the direction of magnetic field is same in multiple brightening loops. Note that the microflares discussed here are fairly large and we can easily identify more than two loop structures in the brightening. With the spectro-polarimeter (SP) data from the SOT, electric current density at the footpoints of brightening loops can be derived from the transverse field information. The electric current density map shows that the electric current is enhanced around the footpoints of brightening loops. Since one end of the brightening loops is located at the sunspot umbra, the current enhancement can be confirmed with high confidence. Assuming that the electric current is aligned along the vertical oriented umbral field, we interpret that magnetic field lines of the brightening loops are twisted. Field discontinuities may be easily formed in the corona, when the field lines are twisted at the base of loops. This suggests energy release among twisted magnetic fields in the corona.

Existence of twisted magnetic fields is one of important factors for causing various kinds of activities in the upper atmosphere. Shimizu et al. (2009) presented one of examples in which twisted fields play key roles in producing remarkable activities in the chromosphere. Long-lasting recurrent ejections of the chromospheric materials were found along a light bridge formed in the sunspot umbra. Ejections were recurrently and intermittently observed for almost 2 days. High-speed upward gas flow was observed in Ca II H images. At the same time, a downward flow signal was observed in the Stokes data measured in the photospheric level, meaning that a bi-directional flow is formed at the low chromosphere or photosphere. One remarkable feature revealed from vector magnetic field data is that the electric current density is significantly enhanced along the light bridge.

From the observations, we interpreted that twisted magnetic flux loop is trapped along the light bridge below the cusp-like magnetic field, as illustrated in Shimizu et al. (2009). Patchy and intermittent reconnections may be exited between the poloidal component of helical twisted loop and vertically oriented umbral fields. However, details of mechanism for producing reconnections intermittently for a long time are unknown and it is important to understand the 3D magnetic field configuration for further understanding. 


\section{Summary}

Most of intense dynamical activities take place in corona and chromosphere above active regions, especially around sunspots. The presentation briefly discussed magnetic origins at the solar surface responsible for causing microflares in corona and transient plasma ejections in the chromosphere. Opposite magnetic polarities encountering each other, created by flux emergence and flux movement, are responsible for many microflares, while about half of microflares do not show such encounters near the microflares. For such cases, twisted magnetic fields, which can be observationally inferred from enhanced electric currents, may be formed in the corona. We need 3D configuration of magnetic fields for the better understanding. Twisted magnetic fields are also key for chromospheric activities, as demonstrated by long-lasting plasma ejections along light bridge.

\section{Acknowledgements}

Hinode is a Japanese mission developed by ISAS/JAXA with NAOJ as a domestic partner, and also with NASA (US) and STFC (UK) as international partners. It is operated by these agencies in corporation with ESA and NSC (Norway). The author would like to express his thanks to the SOC to invite me to present this paper in this symposium.

\section{References}

Aschwanden, M. J. et al. 2000, Astrophys. J., 535, 1047

Kano, R. \& Shimizu, T., Tarbell. T. D. 2010, Astrophys. J., 720, 1136

Shimizu, T. 1995, Pub. Astron. Soc. Jap., 47, 251

Shimizu, T., Shine, R. A., Title, A. M., Tarbell, T. D., \& Frank, Z. 2002, Astrophys. J., 574, 1074

Shimizu, T. et al. 2009, ApJ, 696, L66

\section{Discussion}

Kosovichev: I'd like to comment that in the past this model is the connection of emerging magnetic flux developed by Heyvaerts and Priest. I also have a question. You had this event - the disappearance of a sunspot after a flare. How many events have you observed with Hinode where a sunspot disappears after a flare?

Shimitzu: You mean the sunspot disappear after flare?

Kosovichev: Yeah.

Shimitzu: Actually no. I examined small micro-flares. Its energy is smaller than $10^{27}$ ergs. For such cases we see small magnetic flux as the photospheric counterpart, and such flux is not recognized as a sunspot. OKay? Large flares may be related to large sunspots. I'm not sure whether such complete disappearance of the sunspot can be caused by the occurrence of large flares.

PRIEST: It is very interesting that half of your micro flares are due to this process of either flux cancelation or flux emergence that Karen Harvey and Sarah Martin studied so well many years ago; but the other half, it would be great to study those in more detail because it looks like maybe there is some kind of reconnection process in the 
corona which is occurring spontaneously rather than being driven like the first class. So it would be very interesting to try to understand what kind of instabilities. There are many possibilities theoretically, but it would be interesting to try and study a few of those cases in very great detail.

Shimitzu: Yes. 